definite mental disease, nor if the patient is very fat, nor, in the author's opinion, in cases of paralysis whather of sensation or motion. In further insisting on the difference between true hysteria and malingering, Dr. Hale White mentions the case of a girl of good position who was the subject of an extraordinary rash over nearly the whole of the trunk and limbs. The astual mode of causation was never discovered, but it was ascertained that by encasing a limb in plaster of Paris it was possible to excluds the envaloped limb from the area of eruption. It was quite clear that the girl was a malingerer pure and simple.

1 Clin. Jour., June 7, 1905.

CANCERJUS DISFASE OF THE STOMACH WITH SECONDARY INFECTION THROUGH THE THORACIC DUCT.

IT is an important clinical memorandum that enlargement of the lymphatic glands at the root of the neck may bə an index of malignant disease in the upper part of the alimentary canal. The significance of this rule is all the greater seeing that such enlargement may be the one fact which attracts the patient's attention, whilst dysphagia or other symptoms pointing to the primary growth may be unrecognised. Even a considerable epitheliomatous ulcer of the pharynx ${ }^{1}$ has been known to cause no local complaint, and has only brought the patient under medical observation when there was secondary extension to the cervical glands. The value of the observation may also be illustrated in cases of dysphagia due to œsophageal obstruction. The fast of the obstruction may be determined beyond doubt by the use of a bougie, but there remains the important question, What is the cause of the obstruction ? and in such circumstances cervical glandular enlargement becomes of high clinical importance. In this association considerable interest is attached to a case of "latent" carcinoma of the stomach reported by Dr. W. Mitchell Stevens, ${ }^{2}$ in which an early diagnosis was made from the existence of glandular enlargement in the left supra-clavicular region. The patient, a man of 46 years, presented himself at hospital with œdema of the genitals and legs of about a week's duration. On physical examination the only additional abnormal facts discovered were a moderate painless enlargement of the liver and an enlarged gland under the clavicular insertion of the left sterno-mastoid muscle. The gland was fairly movable but vary hard. There was no evidence of disease in the heart, lungs, or kidneys, and the blood gave evidence of a "secondary", anæmia. From these facts it was argued that in the absence of evidence of disease in the neighbourhood the crrvical enlargement was probably the result of malignant disease within the abdomen where, as physical examination showed, the liver was unduly large. Further, as malignant disease of the liver is usually secondary, it was suggested, as no symptoms or evidence of a primary gro!wth could be detected, that the case was one of latent carcinoma of the stomach, and that the œdema was probably due to the pressure of enlarged (malignant) glands on the large venous trunks within the abdomen. The course of the case appeared to justify the accuracy of thes conclusions for tho hepatic and glandular enlargaments continued to increase and the patient gradually to lose flesh and strength; later, too, gastric pain, vomiting and hæmatemesis were added to the symptoms. At the autopsy the case was found to. bəone of malignant disease of the stomach, the grow th involving the posterior wall, and the condition of the liver and the cervical glands was in exact harmony with the original diagnosis. An interesting demonstration that the route of extension to. the cervical glands was by the thoracic duct was afforded by the extensive malignant involvement of the wall of the duct. The sections of the gland showed a structure identical with the gastric tumour. Dr. Nathan $\mathrm{Raw}^{3}$ records a somewhat similar case in which the primary malignant process was in the pancreas. The patient, a man of 46 years, complained only of general weakness and of a swelling in the left side of the neck. No evidence of disease could be found in any other part of the body, and the condition was regarded as lymphadenoma. Gradually the patient got weaker and ultimately died, when the pancreas, the abdominal glands, and the cervical mass were found to be the sites of scirrhous growth. Here also the lower end of the thoracic duct was involved in the disease. These cases obviously have an important bearing on the discussion regarding the mode in which cancer spreads, and will greatly strengthen the position of those who claim that the thoracic duct plays an important part in the process. Possibly, as Dr. Raw suggests, a careful examination of the thoracic duct in cases of death with apparently primary malignant disease of the cervical lymphatic glands would trace not a few of these to a "latent" malignant growth in the abdomen. In the field of diagnosis, too, these records emphasise the importance of considering carefully the possible meaning of a cervical glandular enlargement. Occurring without explanation, such an event demands the most anxious study of the possibility of malignant disease in som 3 part of the alimentary canal.

1 Polyclinic Jour., vol. v., p. 293. ${ }^{2}$ B*it. Med. Jour., April 29, 1905. 3 Ibid., June 24, 1905.

\section{CHEMICAL EXAMINATION OF THE} GASTRIC CONTENTS.

Dr. W. H. WILlcox ${ }^{1}$ points out the important relationship of this form of examination to early diagnosis, which, in view of th interference, has become an increasingly urgent claim. What is wanted is a method having. scientific accuracy and capable at the same time of ready clinical application. As the result of experiments Dr. Willcox concludes that one or other of the following is the most suitable "test meal." A pint of weak tea (infused only two or three minutes) with the addition of not more than one oz. of milk, and sugar if desired; a pint of thin arrowroot made with water and about two ozs. of milk, sugar being added to taste; or cornflour, thin gruel or other carbohydrate food may be used instead of arrowroot. With one or other of the above fluids a round of thin buttered toast should be taken; this stimulates the gastric glands and also the pyloric sphincter which consequently prevents the too ready passage of the food into the intestine. The 
" test meal" should be given in the early morning before any other food, and should be drawn off after the lapse of one and a half hours. Previous washing out of the stomach is not necessary, but a restricted and light diet should be used for several days in advance. To withdraw the gastric contents after the test meal a long, soft tube should be used with a longer external limb than the part inside the alimentary canal. The syphon action can be started by means of a syringe. The presence of free hydrochloric acid in the stomach contents is of great value in the diagnosis, since the acid is almost invariably absent in carcinoma, whilst it is present in gastric, and also in duodenal ulcer. Those who desire to follow out the actual chemical testing of the stomach contents will find some useful practical directions in Dr. Willcox's paper, but the process of analysis nowadays is usually committed to the clinical laboratory.

1 Lancet, June 10, 1905.

\section{Progress in MEdicine and SuRgery.}

\section{PSYCHIATRY.}

General Paralysis Following Syphilis in Wives.General paralysis in married women, arising after syphilitic infection from the husband, is a rare occurrence, but sufficiently numerous cases have at present been investigated to afford support to the theory that conjugal syphilis (contracted by one from the other partner in married life) is a most potent cause of general paralysis. The subject was first dealt with by Ludwig Acker (1887) and Mendel (1888), both of whom reported cases of general paralysis caused in wives by syphilis contracted from their husbands. Various observers have since then placed similar cases on record, as at present, according to Cullere $^{1}$ (1904) no fewer than 40 undoubted cases are known to medical literature. In a recent publication, ${ }^{2}$ Drs. S. Garnier and A. Santenoise of the Dijon Asylum, give an interesting account of a case of " conjugal general paralysis." The patient was an old woman with a neuropathic family history, her mother having been affected with insane delusions, while a first cousin, on the mother's side, had epilepsy and in adult life developed into a general paralytic of the expansive or grandiose type, $:$ The patient married at 49 years of age, but was separated a year later from her husband, who was then taken to an asylum suffering from general paralysis, and who a few days after admission died of "apoplectiform" attacks. (His father had been a patient in the asylum previously, and had died from cerebral softening and organic dementia, while an uncle was a senile dement, with intercurrent maniacal attacks). Shortly after her marriage (1896) she presented signs of syphilitic infection, namely, roseolous rash, sore throat, and other troubles, and was diagnosed as a case of recent syphilis by Dr. P- of Dijon. She, however, refused treatment, saying "she would not be poisoned with medicines." Eight years later she developed all the typical signs of general paralysisspeech embarrassment, tremulous articulation, inequality and contraction of the pupils, tremors of the tongue and lips, difficulty of locomotion, enfeeblement of memory, dementia and untidy and dirty habits. Paroxysmal apoplectiform attacks ensued, and she died in the course of one of these attacks. The interval between syphilitic infection, and the establishment of general paralysis in this case, was about eight years.

Tanzi's Classification of Insanity. - Professor Tanzi, of Florence, a distinguished alienist physician, ${ }^{3}$ and superintendent of the asylum at Milan has recently published an excellent classifica- tion of the insanities, which is as follows. The basis of the classification is etiological and to some extent symptomatological. The following eight "groups" of insanity are recognised :-

(a). Infantile cerebropathies.

1. Idiocy.

2. Imbecility.

(b). Constitutional neuro-psychoses.

3. Constitutional neurasthenic insanity.

4. Hysterical insanity.

5. Epileptic insanity.

(c). Degenerative anomalies of mind.

6 . Perversion of the sexual instinct.

7. Constitutional immorality.

8. Hereditary weak-mindedness.

9. Paranoia.

(d). Dementia pracox.

10. Hebephrenia.

11. Catatonia.

12. Paranoid forms of d. præcox.

e). Affective psychoses.

13. Mania.

14. Melancholia.

15. Circular insanity.

(f). Toxic insanity.

16. Alcoholic insanity.

17. Morphinism, cocainism.

18. Pellagra.

(g). Insanity of infection and auto-intoxication.

19. Confusional dementia.

20. Uræmic psychosis.

21. Thyroid psychoses.

22. General paralysis.

(h). Cerebropathies of adults.

23. Senile dementia.

24. Traumatic insanity.

25. Cerebro-syphilitic insanity.

26. Apoplectic dementia.

The Psychopathology of Mediums, Seers, and Mystics.-Various observations of importance concerning the pathology of mind in mediums and professional seers have been made the subject of communication to, and discussion recently at, the Société Médico-Psychlogique of Paris. ${ }^{4}$ Dr. Gilbert Ballet and Dr. Monier-Vinard presented a case and said that the man, who was a professional "spirit medium," presented all the characters met with in this class, namely, hyperæsthesia and proneness to develop hallucinations of the senses, especially of sight and hearing. The variety and mobility of such hallucinations were extreme. At first the medium showed slight symptoms indicating a facile tendency to "doubling of personality," which has since become more marked. "His present mental status recalls that of the visionary, Sweedenborg, the seer of planets and stars, and of life in the planets. This 'medium' seems to visit (visually by reason of his hallucinations) the planet Saturn; he sees its seas and lands, and describes in detail the appearance of 